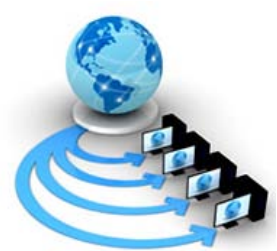

Volume 8, No. 7, July - August 2017

International Journal of Advanced Research in Computer Science

RESEARCH PAPER

\title{
ENERGY EFFICIENT WIRELESS CLUSTERING USING BIOGEOGRAPHY BASED OPTIMIZATION
}

\author{
Anu \\ M Tech. Department of computer science and engineering \\ DCRUST, Sonepat, Haryana
}

\begin{abstract}
Wireless sensor networks are constrained with limited battery lifetime. WSN consist of sensor nodes and cluster heads which are battery operated. hence efficient usage of cluster head energy becomes a crucial factor in the long run operation of a WSN. Many artificial algorithms have been implemented in the past to achieve energy efficient wireless clustering. we propose a new artificial intelligence based wireless clustering algorithm that performs better than all the existing algorithms. We use Biogeography Based Optimization to minimize the energy consumption by cluster heads in a WSN. The proposed algorithm outperforms all the existing algorithms.
\end{abstract}

Keywords: Clustering,Wireless,Sensor, Optimization, Energy

\section{INTRODUCTION}

Wireless sensor networks are becoming increasingly popular with their utility being proved in many areas such as their deployment in battle fields, fighter jets, agriculture, weather and many more. Nature inspired artificial techniques have become popular in recent years and used frequently to solve and optimize many problems including wireless sensor networks. In the past energy efficient wireless clustering are being implemented by using some traditional artificial intelligence technique like genetic algorithm [1], particle swarm optimization [2] [3]. In this paper, we have proposed a new nature inspired evolutionary algorithm BBO to implement efficient wireless clustering in wireless sensor networks.

Wireless sensor networks consist of many small size sensor nodes used to sense useful information. Each node is assigned to a cluster head or gateway. Node sense useful information and transmit this information to the gateway. A gateway receives information from sensor node, aggregate this information and forward it to the sink. This entire scenario forms a cluster in a wireless sensor network [4].

Clustering is important in WSN as it provides a hierarchal, organized and structured schema to collect useful information from various sources and transmit this information to sink through cluster heads. Also, clustering reduces energy consumption in a WSN as all sensor nodes need not to communicate with the sink rather only cluster head communicate with the sink as a representative of all sensor nodes contained in the cluster [4]. In a clustered WSN scenario whole network is divided into cluster heads and sensor nodes out of which a cluster head bears some extra responsibilities like: 1) A cluster head is responsible for aggregating sensed data from its corresponding node. 2) Cluster head is responsible for transmitting this sensed information to the sink. 3). One big problem with WSN is that it is operated by batteries and can operate as long as the battery is alive. Cluster heads in a WSN bear crucial responsibility. Since they are operated by battery, they are constrained by limited energy. Entire cluster goes down as soon as battery power of cluster head is consumed [4]. In WSN implemented in the past cluster heads were chosen out of ordinary sensor nodes that having same energy as any other sensor node. This resulted in the early death of cluster head and hence the early death of the entire cluster. To deal with such problem a special node with some extra energy is proposed as a candidate to be cluster head. Gateways are similar to cluster heads and have same functionalities. They are also battery operated have limited lifetime.

1.2 Author's contributions. As we studied above cluster heads in WSN are operated by battery which is a big hindrance in the efficient transmission of sensed information to the sink. Cluster dies as soon as battery of a gateway is consumed. Gateway with extra energy is introduced, but it is also constrained by battery life time. Hence an efficient clustering algorithm is required to achieve maximum cluster lifetime, even with the use of gateways,

In this paper evolutionary algorithm biogeography based optimization is used for clustering in WSN. BBO was introduced by Dan Simon in 2008 [5]. This is an algorithm similar to genetic algorithm with few changes. In BBO population consists of many habitats as chromosomes in GA. Goodness of a solution is given by habitat suitability index as fitness in GA. Each habitat have immigration and emigration rate based on its HSI value. Habitat with high HSI will emigrate species to habitat having low HSI. In this way migration is performed in BBO which is a main step to maintain diversity in the population. In BBO initial solution is never discarded, it is modified [5] [6] [7] [8].

$\mathrm{BBO}$ is applied in this paper to achieve energy efficient clustering in WSN. Sensor nodes and gateways are taken as habitats. Their HSI value is calculated taking into account 1) Distance of individual node from the gateway. 2) Distance of gateway from sink. 3) Residual energy of gateway. Based on HSI values immigration and emigration rates of habitats are calculated which further leads to migration operation between habitats. Migration operation is done using MPX crossover which increases the diversity in the population. HSI values of modified habitats are recalculated, which showed a drastic improvement from HSI values before 
migration. Finally entire scenario is simulated in Matlab to obtain optimized results.

The proposed BBO based wireless clustering improves the lifetime of a gateway. Its performance is compared with some existing algorithms like PSO based clustering [3], GA based clustering [2], GLBCA [9], LDC [10].

The rest of the paper is organized as follows. The related work is presented in Section 2. The proposed algorithms and the experimental results are presented in Sections 3 and 4 respectively.

\section{RELATED WORK}

Manny approaches have been used in which artificial intelligence techniques are used to improve lifetime of a wireless cluster. In [11] a clustering algorithm is proposed in which a breadth first tree is used to find the gateway which is least loaded and then sensor node is assigned to that gateway. One big problem with this algorithm is it took a large amount of memory space to store and process BFS. Also execution time while calculating BFS is large. It has time complexity of the order $\mathrm{O}\left(\mathrm{mn}^{2}\right)$. In [2] a genetic algorithm based load balancing, clustering algorithm is proposed. Mutation step of genetic algorithm is used for load balancing. In [11] genetic algorithm is used for routing of aggregated data between cluster head and sink. In this algorithm roulette wheel selection is used for selection of individuals and fitness of an individual is taken in terms of network lifetime. In [11] another routing algorithm is proposed in which attempt is made to reduce communication distance between the sink and gateway using genetic algorithm. One thing to be noted in both [11]], only the transmission of aggregated data from cluster head to the base station is focused upon, there is no emphasis on energy consumption in data transfer between individual node to cluster head. In [3] a particle swarm optimization based clustering is proposed to enhance lifetime of a wireless cluster.

Most of above algorithm focus on clustering only and do not take into account routing overhead involved in the clustering process. Also, some of the above algorithms focus only upon energy consumed in sending aggregated data from cluster head to sink while achieving energy efficient clustering, they do not take into account overhead involved in routing from an individual node to cluster head. Our proposed BBO algorithm rectifies these flaws by taking into account 1) Distance between individual node and gateway 2) Distance between gateway and sink 3) Residual energy of the gateway in HSI calculation. To our knowledge, there is no such algorithm existing presently, in which artificial intelligence technique BBO is applied for energy efficient wireless clustering taking into account both individual nodes to gateway routing overhead and gateway to sink routing overhead.

\section{Overview of BBO}

BBO is an evolutionary algorithm based on biogeography based optimization. Biogeography is the way nature distributes species and is analogous to general problem solutions [5]. In past many algorithms like genetic algorithm, particle swarm optimization and ant colony optimization were implemented for obtaining an optimum and fittest solution. BBO is an evolutionary algorithm that is implemented with the migration of species from one habitat to another habitat to maintain diversity in the population. [6].

In $\mathrm{BBO}$ an individual is represented by a habitat. A population may contain many habitats like chromosomes in genetic algorithms. For each habitat in the population, habitat suitability index (HSI) value is calculated. HSI is a measure of the goodness of the habitat or solution. A habitat having high HSI value is considered to have higher fitness or more, suitable for population to grow and vice versa for habitat having low HSI value [7]. Based on this HSI value rank of each individual is calculated. Migration operation is performed to maintain diversity in the population in BBO. Migration involves migration of species from habitat having higher HSI value to habitat having lower HSI value. Migration operation is based on immigration rate and emigration rate. A habitat having high HSI value will emigrate its suitability index variable (SIV) to habitat having low HSI value and habitat having low HSI value will immigrate SIV from habitat having high HSI value [8]. This emigration and immigration are done on the basis of emigration rate (mue) and immigration rate (lambda) which are calculated as follows:

Immigration rate (lambda) $=\mathrm{I}\left(1-\mathrm{k}_{\mathrm{i}} / \mathrm{n}\right)$

Emigration rate $($ mue $)=\mathrm{E}\left(\mathrm{k}_{\mathrm{i}} / \mathrm{n}\right)$

$\mathrm{I}=$ maximum immigration rate.

$\mathrm{E}=$ maximum emigration rate.

$\mathrm{K}_{\mathrm{i}}=$ rank of the habitat.

$\mathrm{n}=$ total number of habitats.

\section{PROPOSED ALGORITHM}

In the proposed work we have considered 36 sensor nodes placed at a fixed location having 6 cluster heads/gateways between them as shown in figure 5.1. We aim to cluster these sensor nodes such that every cluster contains 5 sensor nodes and a gateway. We aim to maximize the lifetime of a wireless cluster. We have used biogeography based optimization tool for wireless clustering and make our cluster last for more number of rounds.

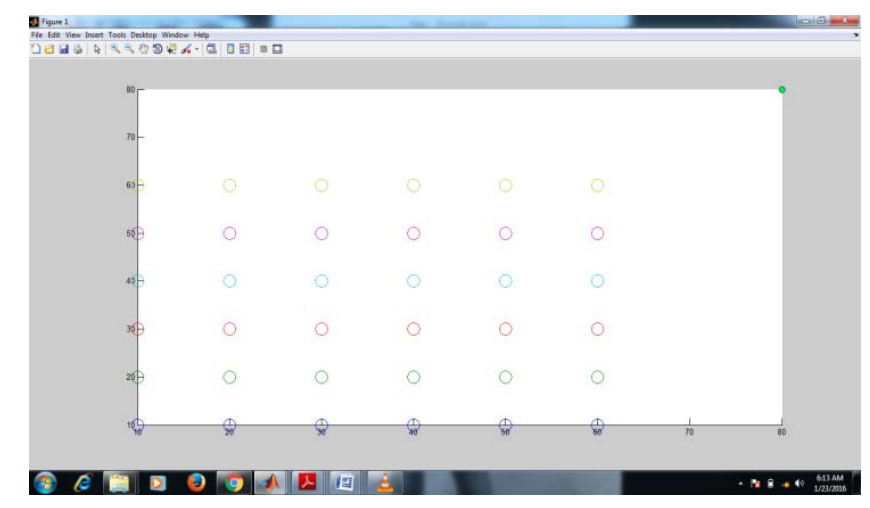

Figure 5.1 - 36 sensor nodes placed at a fixed location with a sink.

5.1 Calculation of HSI. BBO is analogous to the genetic algorithm. In GA we use to calculate the fitness of every chromosome in the population. In BBO we calculate habitat suitability index value of each habitat of the population. The HSI value of a habitat is calculated using the following equation: 
HSI $=\mathrm{k} *$ Energy $(\mathrm{C} . \mathrm{H})+(1 /($ Distance $($ Sink, C.H $)+$ Distance(Node, C.H) ) )

Here $\mathrm{k}=$ constant

Energy $(\mathrm{C} . \mathrm{H})=$ residual energy of cluster head

Distance (Sink, C.H = distance between sink and cluster head.

Distance $($ Node, C.H) = distance between a node and cluster head.

5.2 Biogeography based optimization. Now wireless clustering and performed using $\mathrm{BBO}$ and energy efficient clusters are made using the following technique.

5.2.1 Habitat initialization. 36 sensor nodes are placed at a fixed location. S_N = s1, s2, s3, s4.............s36 $\} .6$ gateways are chosen as $\mathrm{G}=\{\mathrm{g} 1$, g2, g3...g6 $\}$. Initialize habitats in the ecosystem with each habitat containing 2 vectors, one containing sensor nodes from s1 to s36 and another containing corresponding gateways randomly assigned to sensor nodes. The length of both the vectors is same. Entire population contains many habitats like this. Calculate HSI value of every individual gateway in gateway vector of each habitat with following formula:

HSI $=\mathrm{k} *$ Energy $(\mathrm{C} . \mathrm{H})+(1 /($ Distance $($ Sink, C.H $)+$ Distance(Node, C.H) ) )

For every gateway vector of each habitat, take the sum of HSI of all individual gateways. This will give a total HSI value of entire habitat. Now we have all possible habitats and their HSI values. Sort HSI values in increasing order and assign a rank to each habitat such that worse habitat gets the first rank and best habitat gets the last rank.

Rank = sort (HSI value of every habitat)

Subject to constraint $=$ ascending order of sorting.

Above constraint make sure that habitat with higher HSI value does not have a higher immigration rate than habitat having low HSI value. If the best HSI habitat is assigned rank 1 , it will end up with higher immigration rate than the low HSI habitat value according to the formula given in equation 5.3 and 5.4. This may disprove BBO.

5.2.2 Immigration and emigration rates. Emigration and immigration rates are calculated as follows:

Immigration rate (lambda) $=\mathrm{I}\left(1-\mathrm{k}_{\mathrm{i}} / \mathrm{n}\right)(5.3)$

Emigration rate (mue) $=\mathrm{E}\left(\mathrm{k}_{\mathrm{i}} / \mathrm{n}\right)$

$\mathrm{I}=$ maximum immigration rate.

$\mathrm{E}=$ maximum emigration rate.

$\mathrm{K}_{\mathrm{i}}=$ rank of the habitat.

$\mathrm{n}=$ total number of habitats.

Habitats having high HSI value will have a low immigration rate and high emigration rate. SIV will migrate from high HSI value or high emigration rate habitat to the low HSI value of high immigration rate habitat.

5.2.3 Migration operator. A random number $\mathrm{r}$ is generated. For habitat $=i$, If $\mathrm{r}<$ immigration rate (i), choose $\mathrm{i}$ as immigrating habitat. Choose habitat with highest HSI value as emigrating habitat. Make a crossover between these two habitats using MPX. This will result in a modified gateway habitat/vector let corresponding to sensor node vector to which gateways have been assigned now using this modified vector. In this way we will obtain many modified vector/habitat assigned to sensor nodes. Calculate HSI values of all these resulting habitats again. Retain the habitat with best HSI value. If the HSI value after modification is better than the HSI value before modification, a batter network performance is achieved. The whole scenario is then simulated and optimum output is achieved.

\section{RESULTS}

We have used Matlab programming to simulate the proposed algorithm for wireless network. Network performance is tested for 36 nodes, 100 nodes, 200 nodes, 300 nodes 400 nodes, 500 nodes and 700 nodes.

Simulation parameters used are described in table 6.1 and 6.2 .

Table 6.1 - simulation parameters used in the simulation.

\begin{tabular}{|l|l|}
\hline Parameters & Value taken \\
\hline Sensor nodes & $36-500$ \\
\hline Area & $500 * 500 \mathrm{~m}$ sq. \\
\hline Gateways & $6-50$ \\
\hline Initial energy of nodes & 2 Joules \\
\hline Initial energy of nodes & 10 Joules \\
\hline
\end{tabular}

Table 6.2 - BBO parameters used in simulation run.

\begin{tabular}{|l|l|}
\hline Parameter & Value taken \\
\hline Number of habitats & 1000 \\
\hline $\begin{array}{l}\text { Maximum immigration } \\
\text { rate }\end{array}$ & 1 \\
\hline $\begin{array}{l}\text { Maximum emigration } \\
\text { rate }\end{array}$ & 1 \\
\hline $\mathrm{HSI}_{\text {max }}$ & \\
\hline $\mathrm{HSI}_{\text {min }}$ & \\
\hline
\end{tabular}

Comparison of proposed work with existing work -

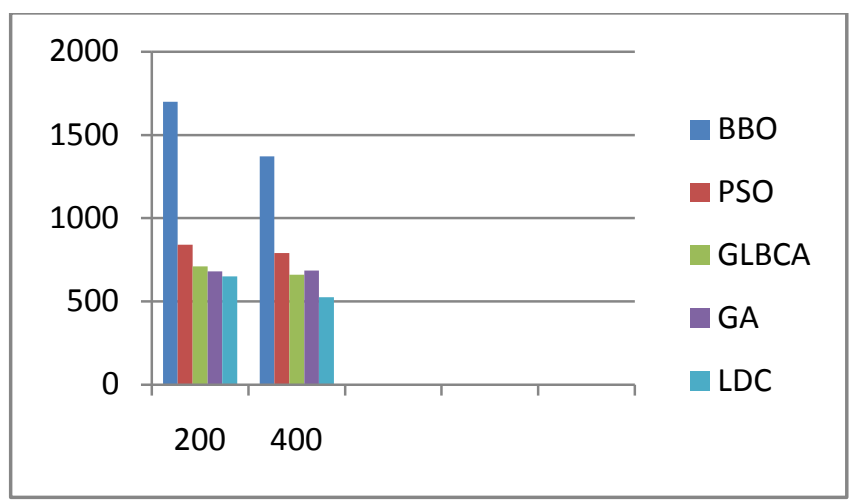

$\mathrm{X}$ axis - Number of rounds.

$\mathrm{Y}$ axis - Number of sensor nodes. 
Figure 6.1 - Output for 200, 400 nodes compared to existing work when distance between nodes is 2.5 , cluster heads are 25 and sink is at distant location

\section{REFERENCES}

[1]. Pratyay Kuila et. Al., A novel evolutionary approach for load balanced clustering problem for wireless sensor networks, Swarm and Evolutionary Computation, p 48-56, 2013.

[2]. Pratyay Kuila et. Al., Energy efficient clustering and routing algorithms for wireless sensor networks: Particle swarm optimization approach, Engineering Applications of Artificial Intelligence, p 127-140, 2014.

[3]. Morteza M. Zanjireh et. Al., A survey on centralized and distributed clustering routing algorithm for WSNs, IEEE, 2015.

[4]. Dan Simon, Biogeography-Based Optimization, IEEE transactions on evolutionary computation, 12 (6), 2008.
[5]. Daya Gupta et. Al., Enhanced Heuristic Approach for Travelling Tournament Problem based on Extended Species Abundance Models of Biogeography, International Conference on Advances in Computing, Communications and Informatics, 2014..6

[6]. Lavika Goel et. Al., Extended Species Abundance Models of Biogeography Based Optimization ,Fourth International Conference on Computational Intelligence, Modelling and Simulation, 2012.

[7]. Seyed Habib A et. Al., A new biogeography-based optimization (BBO) algorithm for the flexible job shop scheduling problem, International Journal Advances in Manufacturing Technology, p 1115-1129, 2012.

[8]. Low, C.P. et. AL., Efficient load-balanced clustering algorithms for wireless sensor networks.,750-759, 2008.

[9]. Ataul et al., Clustering strategies for improving the lifetime of two-tiered sensor networks.,3451-3459, 2008.

[10].Gupta, G.,Younis,M., Load-balanced clustering of wireless sensor networks.1848-1852, 2003.s

[11].Ataul et Al., Agenetica algorithm based approach for energy efficient routing, 665-676, 2009. 